BULL. AUSTRAL. MATH. SOC.

VOL. 9 (1973), 161-168.

\title{
Metrization of spaces having Čech dimension zero
}

\section{K.A. Broughan}

\begin{abstract}
A metrizable topological space has a metric taking values in a closed subset of the real numbers having ${ }^{2}$ ech dimension zero if and only if the space itself has Cech dimension zero. We call a development $D=\left(D_{n}\right)$ for a topological space $(X, T)$ a sieve for $X$ if the sets in each $D_{n}$ are pairwise disjoint. Then a Hausdorff topological space $(X, T)$ has a compatible metric taking values in a closed subset of the real numbers having $\nearrow_{e c h}$ dimension zero if and only if there exists a sieve for $X$.
\end{abstract}

\section{Introduction}

In this paper we prove that a necessary and sufficient condition for a topological space to have a compatible metric taking values in a closed zero dimensional subset of the real numbers is that the space be metrizable and have $\check{C}_{\text {ech }}$ dimension zero. We also prove that the two topological properties Cech dimension zero and metrizable, when taken together, are equivalent to a single topological property - namely, having a development consisting of families of open sets which partition the space. These theorems are an extension of the theorem given in [1]: a metrizable space has $\nearrow_{e c h}$ dimension zero if and only if there exists a metric for the space, compatible with the topology, taking values in some subset of the real numbers with zero as its only cluster point. The proofs of the theorems depend on this characterization.

THEOREM 1. Let $(X, T)$ be a metrizable topological space with a

Received 27 March 1973. 
compatible metric taking values in a closed subset of the real numbers having dimension zero. Then $(X, T)$ has tech dimension zero.

Proof. Regard $(X, T)$ as a metric space with the given metric taking values in a subset $F$ of the real numbers having dimension zero. We may assume that $F$ is contained in a closed bounded interval of the real numbers. Embed $X$ isometrically in a Banach space $(B,\|\cdot\|)$ in such a way that if we identify $X$ with its image in $B$, then $0 \in X$ (see [4]). Then if $x$ and $y$ are in $X,\|x-y\|$ is in $F$.

Now let $H=\{0\} \cup\{1 / n \mid n \in N\}, N$ being the natural numbers. Because $F$ has dimension zero and is a subset of $R$, the real numbers, we can find a metric $\rho$ which is compatible with the topology $F$ inherits as a subspace of $R$, and which takes values in $H$. Then, if $x$ and $y$ are in $X$, let

$$
d(x, y)=\max _{a \in X} \rho(\|x-a\|,\|y-a\|) .
$$

It is clear that $d$ satisfies the triangle law, is symmetric, takes values in $H$ and satisfies $d(x, x)=0$ for all $x$ in $X$. If $d(x, y)=0$ then $\rho(\|x-a\|,\|y-a\|)=0$ for all $a$ in $x$. Let $a=y$. Then, $\rho(\|x-y\|, 0)=0$ and thus $\|x-y\|=0$ which means $x=y$.

To prove $d$ generates the original topology on $X$ assume firstly that $\left\{x_{n}\right\}$ and $x$ are points in $X$ with the property $d\left(x_{n}, x\right) \rightarrow 0$. Then for each $a$ in $x, \rho\left(\left\|x_{n}-a\right\|,\|x-a\|\right) \rightarrow 0$. Let $a=x$. This implies $\rho\left(\left\|x_{n}-x\right\|, 0\right) \rightarrow 0$ and hence, because $\rho$ generates the subspace topology on $F,\left\|x_{n}-x\right\| \rightarrow 0$ in $R$. Conversely assume $\left\|x_{n}-x\right\|+0$. We know that

$$
\left|\left\|x_{n}-a\right\|-\|x-a\|\right| \leq\left\|x_{n}-x\right\| \text { for all } a \text { in } X .
$$

Now regard $\rho$ as a map from $F \times F$ to $H$ with $F$ and $H$ having the standard metric inherited from the real numbers. Because $F$ is compact it follows that $\rho$ is uniformly continuous. In particular we may say that given $\varepsilon>0$ there is a $\delta_{\varepsilon}>0$ such that if $|x-y|<\delta_{\varepsilon}$ then $\rho(x, y)<\varepsilon$ for all $x$ and $y$ in $F$. If $\left\|x_{n}-x\right\|<\delta_{\varepsilon}$ then $\rho\left(\left\|x_{n}-x\right\|,\|x-a\|\right)<\varepsilon$ for all $a$ in $X$ and thus $d\left(x_{n}, x\right) \rightarrow 0$. Thus $d$ 
and $\|\cdot\|$ generate the same topology on $X$.

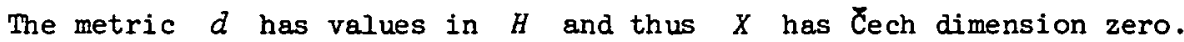
This completes the proof.

From the fact that $H$ is closed and has dimension zero we deduce:

COROLLARY 2. A metrizable space has a compatible metric taking values in a closed subset of $R$ having dimension zero if and only if the space has tech dimension zero.

The proof used in Theorem 1 can be used to prove a more general fact. Let us call a topological space $(X, T)$-metrizable if there is a metric on $X$, compatible with $T$, taking values in $S \subset R$. Suppose further that there exists a continuous metric on $S$ taking values in a subset $T$ of the real numbers and generating a uniform structure on $S$ coarser than that generated by the standard metric inherited from the real numbers. Then if we let

$$
S(T)=\{\sup A \mid \phi \neq A \subset T\},
$$

the method of proof used in Theorem 1 shows that $(X, T)$ is $S(T)$ metrizable.

EXAMPLE 3. On the uniform structures induced by continuous pseudometrics on the rational numbers which take values in $H$.

It is interesting to note that the proof for Theorem 1 does not work for spaces having metrics in dense subsets of the real numbers which have dimension zero (dense at least in a neighbourhood of zero). To see why this is so we will examine the set of all continuous $H$-pseudometrics (that is, having values in $H$ ) on the rational numbers for example.

Let $Q$ denote the rational numbers and let

$$
R(Q)=\{\rho \mid \rho \text { is an } H \text {-metric on } Q \text { generating the usual topology }\}
$$

and $\overparen{R(Q)}=\{\rho \mid \rho$ is a continuous $H$-pseudometric on $Q\}$. If $\rho$ and $\tau$ are in $R(Q)$ let

$$
D(\rho, \tau)=\sup _{(x, y) \in Q^{2}}|\rho(x, y)-\tau(x, y)| .
$$

Then $D$ is a metric and $(R(Q), D)$ a complete metric space. Suppose that $\overparen{R(Q)}$ has the metric topology. 
If $\rho$ and $\tau$ are in $\widehat{R(Q)}$ we will say $\rho \geq \tau$ if $\rho(x, y) \geq \tau(x, y)$ for all $(x, y)$ in $Q^{2}$. Then if $\rho \in R(Q)$ and $\tau \in \overparen{R(Q)}$ and $\tau \geq \rho$, $\tau$ generates a topology on $Q$ finer than that generated by $\rho$, and thus $\tau \in R(Q)$ as it is also continuous.

For each $n$ in $N$, and each $\rho$ in $\widetilde{R(Q)}$ let $\rho_{n}(x, y)=\min \{\rho(x, y), 1 / n\}$. Then if $\rho$ lies in $R(Q)$ so does $\rho_{n}$ and $\rho_{n} \leq \rho$. We see that for this choice of $\rho$ the $D$-limit of $\left\{\rho_{n}\right\}_{n \in N}$ exists and is the zero pseudometric on $Q$. Thus $R(Q)$ is not closed in $R(Q)$. We will show that in fact $R(Q)$ is dense in $R(Q)$.

To this end let $\tau \in \overparen{R(Q)}$ and let $\rho$ be any fixed element of $R(Q)$. (Such an element exists by [1].) Then $\rho \leq \rho \vee \tau$ where $(\rho \vee \tau)(x, y)=\max \{\rho(x, y), \tau(x, y)\}$ and hence $\rho \vee \tau$ is in $R(Q)$ as is $\rho_{n} \vee \tau$ for all $n$ in $N$. We have

$$
\tau=D \text {-limit } \rho_{n} \vee \tau \text {, }
$$

showing that $\overline{R(Q)}=\overparen{R(Q)}$ and that $\widehat{R(Q)}$ is the completion of $R(Q)$. Now suppose that $\rho \in \overparen{R(Q)}$ generates a uniform structure on $Q$ for which the identity $(Q,|\cdot|) \stackrel{i d}{\longrightarrow}(Q, \rho)$ is not uniformly continuous. This means there exists an $\varepsilon_{\rho}>0$ such that for all $n \in N$ there exists $\left(x_{n}, y_{n}\right) \in Q^{2}$ satisfying $\left|x_{n}-y_{n}\right|<1 / n$ and $\rho\left(x_{n}, y_{n}\right) \geq \varepsilon_{p}>0$. Let $K_{n}=\left\{\rho \in \widetilde{R(Q)} \mid\right.$ there is an $\varepsilon_{\rho}$ satisfying the above condition and $\left.\varepsilon_{p} \geq 1 / n\right\}$. Then $K_{1} \subset K_{2} \subset \ldots \subset \widetilde{R(Q)}$. Also if $\rho \in K_{n}$ and $\tau \geq \rho$ then $\tau \in K_{n}$. As $n$ increases the elements in $k_{n} \mathrm{~K}_{n-1}$ become closer to being uniformly continuous. Indeed the set of pseudometrics on $Q$ generating a uniform structure coarser than that generated by the standard metric on $Q$ is given by $\left(U K_{n}\right)^{\prime}=U$ say, where denotes complementation.

Each $K_{m}$ is closed: to see this let $\rho \in K_{m}^{\prime}$. Then there is a $\delta_{m}>0$ such that if $|x-y|<\delta_{m}$ then $\rho(x, y)<1 / m$. Let $\delta=1 / m-1 /(m+1)$ and let $\tau \in B(\rho, \delta)$. This means that for all $(x, y)$ 
in $Q^{2}$,

$$
|\rho(x, y)-\tau(x, y)|<1 / m-1 /(m+1) .
$$

Suppose that $T \in K_{m}$. Then $\forall n \in N$ there exists $\left(x_{n}, y_{n}\right) \in Q^{2}$ with $\left|x_{n}-y_{n}\right|<1 / n$ and $\tau\left(x_{n}, y_{n}\right) \geq 1 / m$. Choose $1 / n \leq \delta_{m}$. Then $1 / m \leq \tau\left(x_{n}, y_{n}\right)<1 / m-1 /(m+1)+\rho\left(x_{n}, y_{n}\right) \leq 1 / m$ as $\rho\left(x_{n}, y_{n}\right) \leq 1 /(m+1)$. This implies $I / m<I / m$, a contradiction. Thus $\tau \in K_{m}^{\prime}$ and $K_{m}^{\prime}$ is open.

The set $K_{m}$ is also open: let $\tau \in K_{m}$ and let $\delta=1 / m-1 /(m+1)$. We will show that $B(\tau, \delta) \subseteq K_{m}$. If $\rho \in B(\tau, \delta)$ then $\sup |\rho(x, y)-\tau(x, y)|<1 / m-1 /(m+1)$. For all $n$ in $N$ there exists $\left(x_{n}, y_{n}\right)$ in $Q^{2}$ such that $\left|x_{n}-y_{n}\right|<1 / n$ and $\tau\left(x_{n}, y_{n}\right) \geq 1 / m$. Then

$$
1 / m \leq \tau\left(x_{n}, y_{n}\right)<\rho\left(x_{n}, y_{n}\right)+1 / m-1 /(m+1) \text {. }
$$

Thus $\rho\left(x_{n}, y_{n}\right)>1 /(m+1)$ which means $\rho\left(x_{n}, y_{n}\right) \geq 1 / m$. Thus $\rho \in K_{m}$ and $K_{m}$ is open.

It follows that $U=\left(U K_{m}\right)^{\prime}$ is closed. Certainly $U \neq \emptyset$ as $0 \in U$, 0 being the zero pseudometric on $Q$. We will show that $U=\{0\}$.

Let $\rho \in U$. Then for all $\varepsilon>0$ there is a $\delta>0$ such that if $|x-y|<\delta$ then $\rho(x, y)<\varepsilon$. Then $(Q,|\cdot|) \stackrel{i d}{\longrightarrow}(Q, \rho)$ is uniformly continuous as is the composite $\left(Q \times Q, \sigma_{n}\right) \stackrel{i d \times i d}{\longrightarrow}\left(Q \times Q, \sigma_{\rho}\right) \stackrel{\rho}{\longrightarrow} H$, where

$$
\sigma_{11}((a, b),(x, y))=|a-x|+|b-y|
$$

and

$$
\sigma_{\rho}((a, b),(x, y))=\rho(a, x)+\rho(b, y) .
$$

Because $(H,|\cdot|)$ is a compact and hence complete uniform space we may extend the map $\rho \circ(i d \times i d)$ to a continuous map from $R \times R$ to $H$. But then connectedness shows that we must have $\rho \equiv 0$. Thus $U=\{0\}$ as claimed. 
Therefore $U \cap R(Q)=\varnothing$ and we may not extend Theorem $I$ to metric spaces with metrics taking values in $Q$ or for that matter any dense Čech dimension zero subset of the real numbers.

Thus $\overparen{R(Q)}=\{0\} \cup \bigcup_{n=1}^{\infty} K_{n}$ and $R(Q) \subset \bigcup_{n=1}^{\infty} K_{n}$. If $\rho \in R(Q)$ then $\rho_{n} \in R(Q) \cap K_{n-1}^{\prime}$ and therefore $R(Q)$ is not contained in any finite union of the $K_{m}^{\prime}$ s.

To conclude this example we see that the metric $D$ has values in the subset

$$
\left\{1 / n_{1}-1 / n_{2} \mid n_{1}, n_{2} \in N \text { with } n_{1} \leq n_{2}\right\} \cup H \text {, }
$$

of the real numbers. It is easy to see that this set is closed and has dimension zero. Thus, by Theorem $1, \overparen{R(Q)}$ has Cech dimension zero.

Let $(X, T)$ be a topological space and let $D=\left(D_{n}\right)_{n} \in N$ be a development for $T$. If the sets in each open cover $D_{n}$ are disjoint we say that $(X, T)$ is sievable and call the family $D$ a compatible sieve for $X$.

THEOREM 4. Let $(X, T)$ be a Hausdorff topological space. Then $(X, T)$ is sievable if and only if $i t$ is metrizable and has $\bar{C}$ ech dimension zero.

Proof. Let $(X, T)$ have a compatible sieve $D=\left(D_{n}\right)$. For each pair $(x, y)$ in $x^{2}$ with $x \neq y$ let

$$
\rho(x, y)=\min \left\{1 / n \mid x, y \in D \in D_{n}\right\} .
$$

If $x=y$ set $\rho(x, y)=0$. Then $\rho$ is an $H$-metric on $X$ generating the topology $T$. Therefore $(X, T)$ is metrizable and, by [1], has Čech dimension zero. Conversely let $(X, T)$ be metrizable and have Cech dimension zero. Then we may embed $X$ in a countable product of discrete spaces, $B(m)$, where $T$ has weight $m$. Because the property "sievable" is hereditary we need only show that $B(m)$ has this property. To do this we will define a compatible sieve on $B(m)$.

For each $n$ in $N$ let $A_{n}$ be a space of cardinality $m$ having the 
discrete topology. Then

$$
B(m)=\prod_{n=1}^{\infty} A_{n}
$$

Let $D_{0}=B(m)$ and for $n=1,2, \ldots$ let

$D_{n}=\left\{\prod_{j=1}^{\infty} B_{j} \mid B_{j}=A_{j}\right.$ for all but $n$ subscripts $j$ in which case

$$
\left.B_{j}=\left\{b_{j}\right\} \text { with } b_{j} \text { in } A_{j}\right\}
$$

Then $D=D_{0} \cup\left(D_{n}\right)_{n \in N}$ is a compatible sieve for $B(m)$. This completes the proof of the theorem. The reader might note that this result follows also from the results of Morita [5].

EXAMPLE 5. The example of Roy [6] of a metrizable space with small inductive dimension zero and $\chi_{e c h}$ dimension $l$ is an example of a metrizable space with small inductive dimension zero which is not sievable.

COROLLARY 6. Sievable spaces are clopen-parasompact [3] and thus admit harmonic partitions of unity [2].

\section{References}

[1] K.A. Broughan, "A metric characterizing Cech dimension zero", Proc. Amer. Math. Soc. (to appear).

[2] K.A. Broughan, "Clopen-paracompact topological spaces", (to appear).

[3] K.A. Broughan, "Dimension zero", (to appear).

[4] Casimir Kuratowski, "Quelques problèmes concernant les espaces métriques non-séparables", Fund. Math. 25 (1935), 534-545.

[5] Kliti Morita, "A condition for the metrizability of topological spaces and for n-dimensionality", Sci. Rep. Tokyo Kyoiku Daigaku Sect. A 5 (1955), 33-36. 
[6] Prabir Roy, "Failure of equivalence of dimension concepts for metric spaces", Bull. Amer. Math. Soc. 68 (1968), 609-613.

Department of Mathematics, University of Waikato,

Hami Iton,

New Zealand. 